\title{
The Dyer-Roeder relation in a universe with particle production
}

\author{
M. de Campos ${ }^{1}$ and J. A. Souza ${ }^{2}$ \\ 1 Physics Department, UFRR, Campus do Paricarãna, Boa Vista, RR., Brasil \\ e-mail: campos@dfis.ufrr.br \\ ${ }^{2}$ UFF Physics Institute, Av. Litorânea s/n, Niterói, RJ., Brasil \\ e-mail: joseuff@bol.com.br
}

Received 16 September 2002 / Accepted 23 March 2004

\begin{abstract}
We have obtained analytical exact solutions of the Dyer-Roeder equation in a cosmological model where creation of particles occurs at the expense of the gravitational field. We discuss the influences of inhomogeneities in the path of a light beam on the apparent diameter of astrophysical objects and consider both redshift independent and redshift dependent distributions of the inhomogeneities.
\end{abstract}

Key words. cosmology: miscellaneous - cosmology: theory

\section{Introduction}

Cosmology has been, for a long time, a fertile ground for speculation. The choice between competing theories was very difficult due to the small amount of reliable experimental data.

Things have changed, however, in the last decades. The quantity of experimental results relating to the age of universe, its expansion and its matter distribution, as well as gravitational lens occurrence statistics and related subjects, has grown to such a extent that the room left today for speculative reasoning in Cosmology has been considerable reduced.

Among the most interesting recent results, are the supernova IA type data, obtained at the end of the 1990s, which gave support to the hypothesis that our universe has an accelerated expansion (Riess 1998; Perlmuter 1998).

These observations lead to a revival of the cosmological constant, as well as to new proposals for candidates able to generate a negative pressure (Caldwell et al. 1998; Peebles \& Vilenkin 1998), for example, quintessence.

According to some of these hypothesis, the universe would have, beyond its usual baryonic matter content and dark matter, also a negative pressure-generating content, a kind of dark energy that represents the vacuum contribution (Peebles \& Ratra 2002; Carrol 2001; Sahni \& Starobinsky 2000).

One of the attractive features of the hypothesis of particle production is that it therefore relates the large-scale properties of the universe to atomic phenomena (Mac Crea 1951). On the other hand, the introduction of this new component for the cosmological fluid gives not only an explanation for the cosmological acceleration, but also eliminates the age problem of the universe, which in the standard model is smaller than the one obtained for the age of the globular clusters. The estimate for the age of the universe depend upon the value of the Hubble constant. If the value of $H_{0}$ is near the upper limit obtained by Freedmann et al. $\left(H_{0}=80 \mathrm{~km} \mathrm{~s}^{-1} \mathrm{Mpc}^{-1}\right)$ (Freedman et al. 1997) and considering the usual standard model $\left(H=\frac{2}{3 t}\right)$, we get some "relief" for the age problem, although not a definitive solution (Ozer 1999).

The model with particle production (OSC) provides also a reasonable fit with respect to kinetic tests, like luminosity distance, angular diameter and the number counts of galaxies versus redshift relation (Alcaniz \& Lima 1999; Lima \& Alcaniz 1999) and in the radiation-dominated era (photon creation) the model can be compatible with present day isotropy and the spectral distribution of Cosmic Microwave Background Radiation (Lima 1997).

The inclusion of $\Lambda$ solves the age of the universe puzzle, but at the expense of creating a new one, the so-called cosmological constant problem (Weinberg 1989). The conciliation between a very large value for this constant, predicted by quantum field theory, and a small one or zero, can be obtained if we consider the cosmological term time-dependent or quintessence models. In spite of, these models cannot explain why the dark energy density is comparable with the matter one.

As an alternative model for the universe, we can introduce a cosmological particle production term, resulting in a scenario that can mimic the effects generated by the inclusion of $\Lambda$. The physics involved is, nevertheless, quite different.

In this work we are going to study the exact solutions of the Dyer-Roeder equation, considering a homogeneous and isotropic universe where particle production occurs at the expense of gravitational field energy (Prigogine 1989; Lima et al. 1996; Waga \& Arcuri 1994; Gariel \& leDenmat 1990; Zindhal 1993; Lima \& Alcaniz 1999; Alcaniz \& Lima 1999).

In Sect. 2 we outline the cosmological model and the relation between the source of particle production and the cosmological term; in Sect. 3 we examine the observational 
constraints for the model with respect to the age of the universe, the acceleration of its expansion, and make some considerations about primordial nucleosynthesis and structure formation, obtaining a narrower range for the values of the creation particle parameter; in Sect. 4 we obtain the Dyer-Roeder equation for a universe with particle production and find exact solutions considering both a redshift independent and redshift dependent distribution of inhomogeneities.

\section{The model}

\subsection{Field equations}

In this section we give a summary of the cosmology with particle production (Prigogine 1989), show how this model mimics the universe with a $\Lambda$ term and find constraints in the creation parameter using the age of the universe and the deceleration parameter.

The universe will be considered homogeneous and isotropic, described by the FRW line element

$\mathrm{d} s^{2}=\mathrm{d} t^{2}-R^{2}(t)\left[\mathrm{d} r^{2}+r^{2} \mathrm{~d} \theta^{2}+r^{2} \sin ^{2}(\theta) \mathrm{d} \phi^{2}\right]$.

The model with particle production is obtained from the field equations

$8 \pi G \rho=3 \frac{\dot{R}^{2}}{R^{2}}+3 \frac{\kappa}{R^{2}}$,

$8 \pi\left(P_{\text {th }}+\tilde{P}\right)=-2 \frac{\ddot{R}}{R}-\frac{\dot{R}^{2}}{R^{2}}-\frac{\kappa}{R^{2}}$,

coupled to the balance equation for the particle number density

$\dot{n}+3 \frac{\dot{R}}{R} n=\Psi$.

Here the dot means time derivative, $\rho$ is the energy density, $P_{\text {th }}$ is the thermodynamical pressure, and $n$ and $\Psi$ are respectively the particle number density and the source of the particle production. $P_{\mathrm{c}}$ is the creation pressure, given by (Calvão et al. 1992)

$\tilde{P}=-\frac{\rho+P_{\mathrm{th}}}{3 n H} \Psi$,

when the particle production is considered as adiabatic process.

Combining Eqs. (2)-(4) with the state equation $P_{\text {th }}=(v-1) \rho$, it follows that

$R \ddot{R}+\left(\frac{3 v-2}{2}-\frac{v \psi}{2 n H}\right)\left(\dot{R}^{2}+\kappa\right)=0$,

where $v$ is a constant and $\kappa$ is the curvature.

For a complete description of the cosmological environment we must have an explicit expression for the source of particle production. Following Lima et al. (Lima et al. 1996), a physically reasonable expression for the particle creation rate is $\Psi=3 n \beta H$. The creation parameter $\beta$ is generally a function of the cosmic era, or equivalently of the parameter $v$. We can assume two different parameter creations, $\beta_{v}$ and $\beta_{\mathrm{m}}$, for radiation and matter eras, respectively (Alcaniz \& Lima 1999). However, since we study a distance treatment in the actual matter dominated phase $(v=0)$, we supposed $\beta_{\mathrm{m}}=\beta$, with $\beta$ constant.
Beyond this, $\beta$ must be positive, otherwise the second law of thermodynamics $\left(S_{; \alpha}^{\alpha}>0\right)$ is violated.

Substituting the particle production rate above in Eq. (6) and integrating, we obtain

$R=R_{0}\left(\frac{t}{t_{0}}\right)^{\frac{2}{3 v(1-\beta)}}$,

where the subscript 0 refers to the present time.

The OSC can mimic a universe with a $\Lambda$ term. To clarify this relation we take into account the entropy production in both cases. For OSC we have

$S_{; \alpha}^{\alpha}=n \dot{\sigma}+\sigma \psi=-\frac{\mu \psi}{T}$

while for a universe with a $\Lambda$ term

$S_{; \alpha}^{\alpha}=-\frac{\dot{\Lambda}}{8 \pi G T}$

$T$ is the temperature, $\mu=\frac{\rho+P}{n}-T \sigma$ is the chemical potential and $\sigma$ is the entropy per particle.

The two scenarios can explain the entropy content of the universe, which is poorly explained using only Einstein's field equations, which are invariant under time reversal (Prigogine 1989).

Considering the process to be adiabatic $(\dot{\sigma}=0)$ and equalizing the specific entropy due to the $\Lambda$ term and due to particle production we obtain

$\frac{\dot{\Lambda}}{8 \pi G} \propto \frac{\rho \psi}{n}$.

For $\psi=3 n \beta H$ this implies $\Lambda \propto t^{-2}$, for a pressureless universe. The two scenarios have identical time dependence for the scale factor, and consequently for the Hubble function, too.

\subsection{Observational constraints}

\subsubsection{Age of the universe}

Using the scale factor (7) the Hubble function is given by

$H=\frac{2}{3}[(1-\beta) t]^{-1}$.

The results from the Hubble Space Telescope Key Project (Freedman 2000) indicate,

$H_{0}=72 \pm 8 \frac{\mathrm{km}}{\mathrm{Mpc} \mathrm{s}}$

for the Hubble constant.

The High $Z$ Supernovae estimates for the age of the universe is $14.2 \pm 1.7$ Gyrs (Riess 1998) and $14.9 \pm 1.4$ Gyrs (Perlmuter 1998). Carreta et al. (2000) using results from Hipparcos, RRLyrae and Chepheids to re-calibrate the globular cluster distance scale, found $12.9 \pm 2.9$ Gyrs. Butcher (1987) used the abundance of the $\mathrm{Th}^{32}$ to estimate the age of the stars and consequently a lower limit for the age of the universe. The interest in Th abundance is related to its half life, about $14.05 \mathrm{Gyr}$, an interesting time from a cosmological point of view. Using the relation between the abundance of Th 
and Eu, Westin et al. (2000) estimate for the universe an age of 15.0 Gyr, while Johnson and Bolte (2000) found 11.4 Gyr. Recently Krauss \& Chaboyer (2000) estimates a lower limit on the age of the universe, 11 Gyr with $95 \%$ confidence.

These values differs substantially from each other, depending on the technique used; we update the interval for the value of the $\beta$ parameter found by Lima et al. (Lima et al. 1996).

Using Eq. (8), the upper limit for $H_{0}$ from Freedman et al. and the estimated age for the oldest objects in our galaxy $(16.3 \mathrm{Gyr})$, we obtain the value 0.49 for the upper limit for the creation parameter $\beta$. To obtain the lower limit for $\beta$, we use the same expression (8), but with the lower limit for $H_{0}$, again from Freedman, and the smallest age for the local oldest stars from Carreta et al. (2000), giving 0.02.

So, the validity interval for the creation parameter is

$0.02<\beta<0.49$.

\subsubsection{Acceleration of the universe}

According to data from type Ia supernovae observations, the universe is accelerating. Generally, this acceleration is thought to be due to repulsive gravity, which can be introduced via a negative pressure, a cosmological term or a quintessence component in the matter content of the universe.

An alternative view would be to consider particle creation, which naturally redefine the energy-momentum tensor and could account for this increasing expansion velocity (Freaza et al. 2002; Alcaniz \& Lima 1999; Lima et al. 1996; Zindhal 2001).

Taking into account Eq. (6), we can write the deceleration parameter in terms of the particle creation source, namely

$Q=\frac{1}{2}-\frac{4 \pi m G \Psi}{H^{3}}$,

where $m$ is the rest mass of the produced particles, and we consider a null value for the curvature. We obtain an accelerated universe, expanding with the scale factor (7), if $\beta>\frac{1}{3}$. Taking into account the age of the oldest objects in our galaxy, the estimate for the Hubble constant and the indications that we live in an accelerating universe, we obtain that

$0.33 \leq \beta \leq 0.49$.

\subsection{Primordial nucleosynthesis}

In spite of the cosmological consequences, the current matter creation rate, $\Psi_{0} \approx 10^{-16}$ nucleons $\mathrm{cm}^{-3} \mathrm{yr}^{-1}$, is nearly the same rate predicted by the steady-state Universe (Hoyle 1993) taking into account $\kappa=0$. This matter creation rate is presently far below detectable limits (Alcaniz \& Lima 1999). Consequently, this indicates, probably, that the early picture of the OSC differs slightly from the standard model with respect to the primordial nucleosynthesis. However a quantitative analysis with a multifluid description is necessary to more accurately determine the primordial nucleosynthesis in OSC.

Considering primordial nucleosynthesis in its standard form with 3 neutrinos flavours, the main parameter of the theory is the baryon number density quoted as its ratio to the number density of photons, namely $\eta=\frac{n_{\mathrm{b}}}{n_{\gamma}}$. Note that, in a given era of evolution of the universe, the model with cosmological particle production affects the number density of photons in the same way that the number density of the baryons. So, in this model $\eta$ does not suffer sensitive variations in relation to the standard model.

\subsection{Growth of structures}

In the OSC model the particles are created from the gravitational field energy. In this case, a negative extra pressure appears in the energy momentum tensor. This extra pressure is responsible for cosmic acceleration and has the same influence as the negative pressure that appears in models with a cosmological term. So, in the same way that the effects of a cosmological term on linear perturbation theory over short distances can be studied using the expression

$\ddot{\delta}+2 H \dot{\delta}-4 \pi G \rho \delta=0$,

we consider that this equation is also applicable to the OSC model, where $\delta$ is the density contrast. Substituting the scale factor (7) in the equation for the density contrast evolution we obtain the following modes

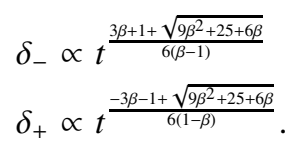

For $\beta=0$ we obtain the usual growing and decaying modes for the dust Friedmann model, $\delta_{+} \propto t^{\frac{2}{3}}, \delta_{-} \propto t^{-1}$, respectively. Note that the $\delta_{+}$for the OSC model grows faster than the growing mode without particle production $(\beta=0)$, including for accelerated expansion of the universe. The few indications in this section are, from our point of view, sufficient to motivate a more detailed study of the scalar density perturbations to furnish a more precise picture of the growth of structures in the OSC model.

\section{The Dyer-Roeder equation}

If there is matter distribution along a cone defined by the bundle of light rays connecting the source to observer, the angular diameter distance of that source from the observer is smaller than that which would occur if the source was seen through an empty cone. This effect is known as Ricci focusing. Our intention in this section is to find and solve the mathematical expression of this effect, the Dyer-Roeder equation, when the particle number in the universe is not conserved.

For a conformally flat metric the Ricci focalization equation is

$\ddot{\sqrt{A}}+\frac{1}{2} R_{\alpha \gamma} k^{\alpha} k^{\gamma} \sqrt{A}=0$,

where the dot means derivative with respect to the affine parameter, $A$ is the beam cross sectional area and $k^{\alpha}=\frac{\mathrm{d} x^{\alpha}}{\mathrm{d} v}$ is the vector tangent to the photon trajectory (Demianski 2000). Considering an energy momentum tensor of the form

$T_{\mu v}=(\rho+P) u_{\mu} u_{v}+P g_{\mu \nu}$, 


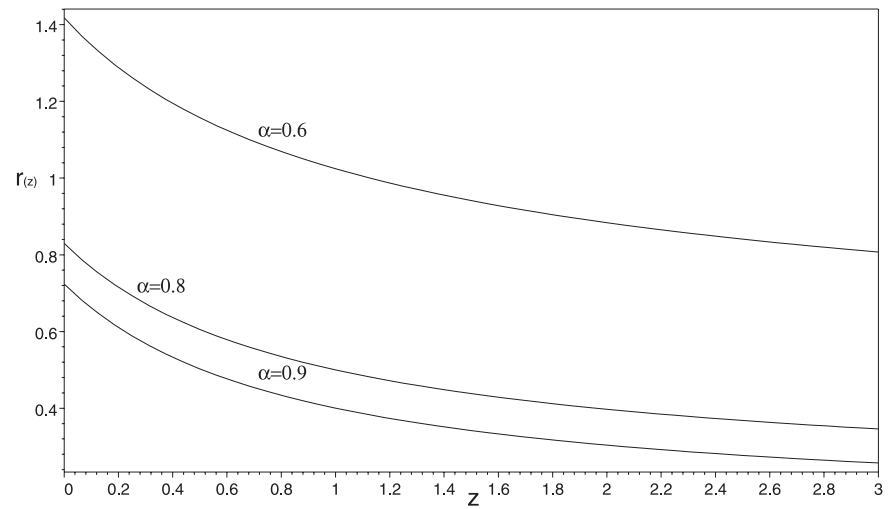

Fig. 1. Evolution of Dyer-Roeder distance for $\beta=0.1$, and three different values of $\alpha$.

where the total pressure $P$ includes contributions from the thermodynamical and creation pressures, we obtain, after straightforward algebra (Giovi et al. 2000)

$\ddot{\sqrt{A}}+4 \pi \frac{\rho+P}{H_{0}^{2}}(1+z)^{2} \sqrt{A}=0$.

Taking into account Eqs. (5) and (7), we obtain, after a simple but a lengthy manipulation (Giovi et al. 2000), the Dyer-Roeder equation in the cosmological background with particle production

$(1+z)^{5} \frac{\mathrm{d}^{2} r}{\mathrm{~d} z^{2}}+\frac{7}{2}(1+z)^{4} \frac{\mathrm{d} r}{\mathrm{~d} z}+\frac{3}{2}(1-\beta)[1+z]^{3(1-\beta)} \alpha r=0$,

where we have defined the dimensionless distance $r=D H_{0}$, $D$ as the angular diameter distance. The parameter $\alpha$ is related to the Ricci focusing and conceptually is the fraction of homogeneously distributed matter inside the light beam (Dyer \& Roeder 1974).

Let us consider two interesting particular cases.

\subsection{Constant $\alpha$}

We recall that, for $\alpha=0$, our model represents a universe where all matter is clustered, while, for $\alpha=1$, we have a universe with its matter content homogeneously distributed.

Equation (14) can be analytically solved for any value of $\alpha$ in the interval from zero to one. We obtain

$r(z)=(1+z)^{-5 / 4}\left\{C_{1} J_{\frac{t 5}{6 \beta}}[\Delta]+C_{2} Y_{\frac{ \pm 5}{6 \beta}}[\Delta]\right\}$,

where

$\Delta=\left(\frac{2 \alpha(1-\beta)}{3 \beta^{2}}\right)^{\frac{1}{2}}(1+z)^{\frac{-3 \beta}{2}}$.

$J$ and $Y$ are the first and second Bessel functions, respectively.

The profile for $r(z)$ when the universe is non accelerated $\left(\beta<\frac{1}{3}\right)$ is shown in Fig. 1.

For an accelerated universe $\left(\beta>\frac{1}{3}\right)$ the profile is the one seen in Fig. 2. To obtain the limiting case when there is no particle production we must put $\beta=0$ in the original differential Eq. (14).

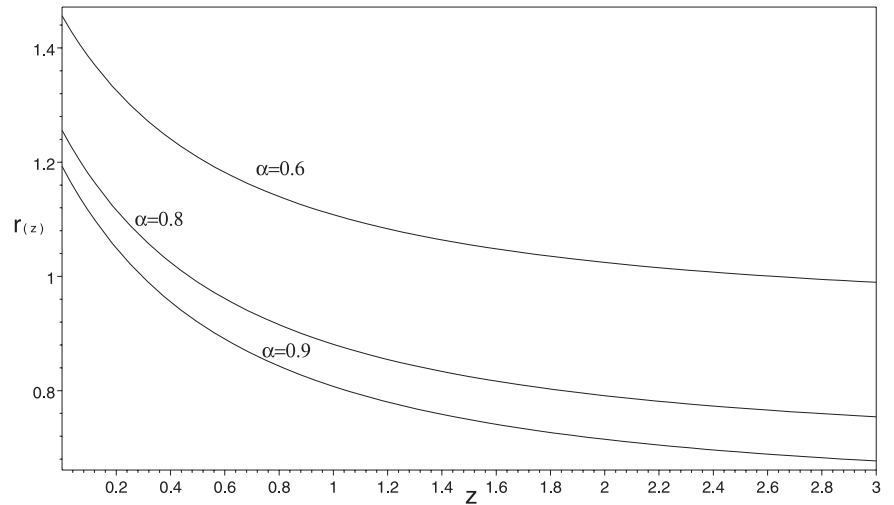

Fig. 2. Evolution of Dyer-Roeder distance for $\beta=0.4$, and the same three different values of $\alpha$.

\subsection{Variable $\alpha$}

In this section we solve the Dyer-Roeder equation for the case where $\alpha$ is no longer a constant, but instead a particular function of the redshift. We adopt for $\alpha(z)$ an expression identical to the one used by Linder (1998), namely

$\alpha(z)=p(1+z)^{q}$,

where $p$ and $q$ are constants. It is reasonable to expect the clumping to decrease with increasing redshift, implying $q>0$. Some care is needing in choosing the parameters $p$ and $q$ is due to the range of $\alpha$, that must stay in the interval $0 \leq \alpha \leq 1$. Naturally, this will depend on the range of redshift studied.

Integration of Eq. (14) now results in

$r(z)={ }_{-} C l J_{A}(B)+{ }_{-} C 2 Y_{A}(B)$,

where

$A=\frac{5}{2(5-3 \beta+q)}$

and

$B=\frac{\sqrt{6(1-\beta) p}(1+z)^{5 / 2-3 / 2 \beta+1 / 2 q}}{5-3 \beta+q}$.

The profile of $r(z)$ in this case, is given by Fig. 3, for specific values of $q$ and $p$, when the universe is not accelerated $\left(\beta<\frac{1}{3}\right)$.

For an accelerated universe $\left(\beta>\frac{1}{3}\right)$ the profile is illustrated in Fig. 4.

\section{Conclusions and final remarks}

The current matter creation rate, $\Psi_{0} \approx 10^{-16}$ nucleons $\mathrm{cm}^{-3} \mathrm{yr}^{-1}$, is nearly the same rate predicted by the steadystate universe (Hoyle 1993), regardless of the value of the curvature parameter. In spite of the matter creation rate presently being far below detectable limits (Alcaniz \& Lima 1999), the physical consequences for the evolution of the universe are potentially measurable.

We have updated (and narrowed) the range of allowed values for the creation parameter $\beta$. 


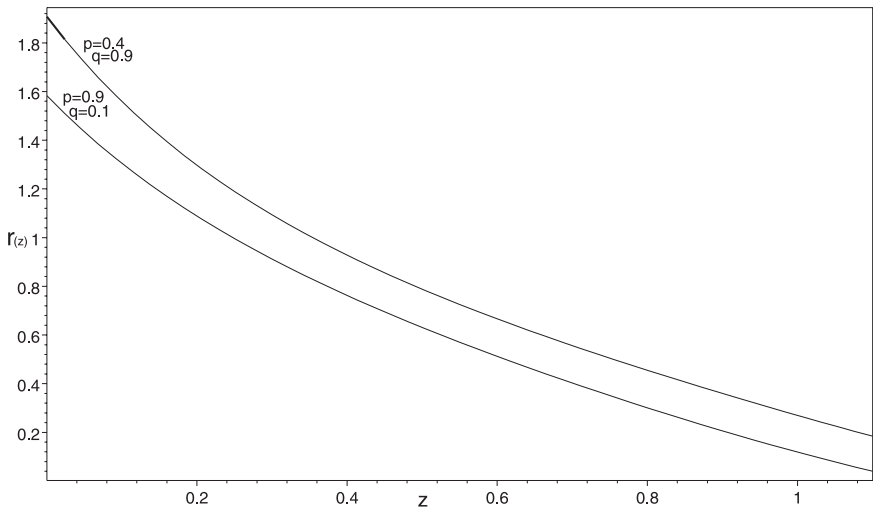

Fig. 3. Evolution of the Dyer-Roeder distance for $\beta=0.25$, and two different values of $q$ and $p$.

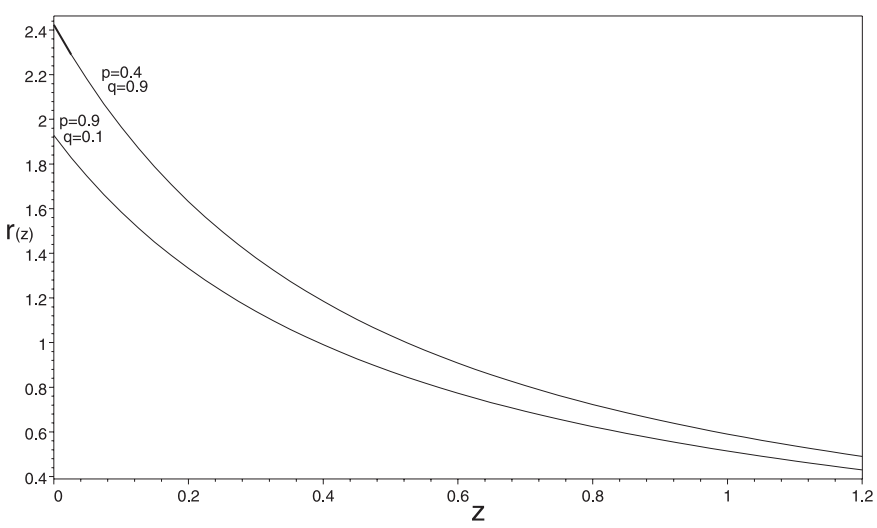

Fig. 4. Evolution of Dyer-Roeder distance for $\beta=0.8$, and same two different values of $q$ and $p$.

For the Dyer-Roeder equation, we have solved it in a background universe with particle production, expanding considerably the manifold of solutions. As far as we know, this equation has previously been solved only for some special constant values of $\alpha$ (Giovi et al. 2000).

In Fig. 1 we have plotted $r(z)$ for a non accelerated universe, for three different values of constant $\alpha$. We note that increasing the inhomogeneities in the path of the light beam (smaller $\alpha$ ) leads to greater values for the Dyer-Roeder distance at the same redshift. Remembering the definition of $r(z)$, this implies smaller values for the viewing angle as $\alpha$ diminishes.

We can interpret these results saying that the objects will appear at apparent distances greater than the ones we would observe in a totally homogeneous universe $(\alpha=1)$.
In Fig. 2 we repeat the same graph, but now in an accelerated universe. We observe the same qualitative behavior, with little enhancing of the effect. The origin of this effect is in the inhomogeneities, while the acceleration, although having some influence, is not the main agent in this case.

In Figs. 3 and 4 we show $r(z)$ in the case of variable $\alpha$, given by Eq. (19), for non accelerated $(\beta=0.25)$ and accelerated $(\beta=$ 0.8 ) universes, for different values of $q$ and $p$. In both cases, for the redshift interval studied, the range of the smoothness function $\alpha(z)$ lies in the interval $0 \leq \alpha(z) \leq 1$.

The next steps for future work are to analyze the lensing probability occurrence for OSC.

\section{References}

Alcaniz, J. S., \& Lima, J. A. 1999, A\&A, 349, 729

Caldwell, R. R., Dave, R., \& Steinhardt, P. J. 1998, Phy. Rev. Lett., 80,1582

Calvão, M. O., Lima, J. A. S., \& Waga, I. 1992, Phys. Lett. A, 162, 223

Carrol, S. 2001, Living Rev. Rel., 4, 1

Demianski, M., Demiansk, R., de Ritis, A. A., Marino, et al. 2000 [arXiv: astro-ph/0004376]

Dyer, C. C., \& Roeder, R. 1974, ApJ, 189, 167

Freaza, M. P., de Souza, R. S., \& Waga, I. 2002, Phy. Rev. D, 66, 103502

Freedman, W. L., Madore, B. F., \& Kennicut, R. C. 1997, The Extragalactic Distance Scale ed. M. Donahue, \& M. Livio

Freedman, W. L., Freedman, B. F., Madore, B. K., Gibson, et al. 2000 [arXiv:astro-ph/0012376]

Gariel, J., \& leDenmat, G. 1990, Phys. Lett. A, 170, 373

Giovi, F., Occhionero, F., \& Amendola, L. 2000, MNRAS, 325, 1097

Hoyle, F., Burbidge, G., Narlikar, J. V., et al. 1993, ApJ, 410, 437

Lima, J. A. 1997, Gen. Relat. Grav., 29, 805

Lima, J. A. S., \& Alcaniz, J. S. 1999, A\&A, 348, 1

Lima, J. A. S., Germano, A. S., \& Abramo, L. R. W. 1996, Phys. Rev. D, 53, 4287

Mac Crea, W. 1951, Proc. R. Soc. London A, 206, 562

Ozer, M. 1999, ApJ, 520, 45

Peebles, P., \& Ratra, B. 2002 [arXiv: astro-ph/0207347]

Peebles, P., \& Vilenkin, A. 1998 [arXiv: astro-ph/9810509]

Perlmuter, S., et al. 1998, ApJ, 517, 565

Prigogine, I., et al. 1989, Gen. Relat. Grav., 21, 767

Riess, A. G., et al. 1998, ApJ, 116, 1009

Sahni, V., \& Starobinsky, A. 2000, Int. J. Mod. Phys. D, 9, 373

Waga, I., \& Arcuri, R. C. 1994, Phys. Rev. D, 50, 2928

Weinberg, S. 1989, Rev. Mod. Phys., 61, 1

Zindhal, W., et al. 1993, Class. Quantum Grav., 10, 1775

Zindhal, W., et al. 2001, Phys. Rev. D, 64, 063501 